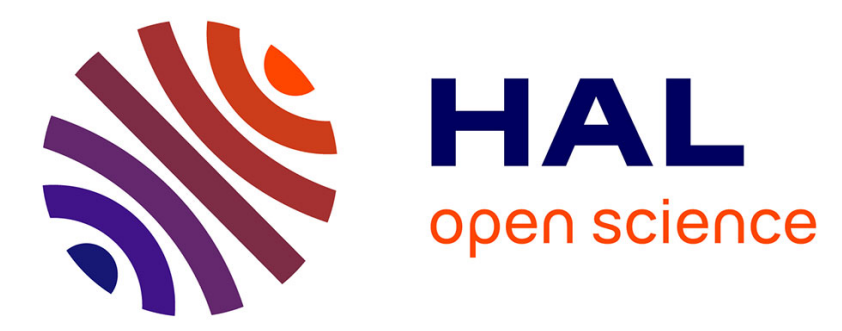

\title{
Field mapping measurements to determine spatial and field dependence of critical current density in YBCO tapes
}

\author{
Julien Leclerc, Kévin Berger, Bruno Douine, Jean Lévêque
}

\section{To cite this version:}

Julien Leclerc, Kévin Berger, Bruno Douine, Jean Lévêque. Field mapping measurements to determine spatial and field dependence of critical current density in YBCO tapes. Physica C: Superconductivity and its Applications, 2013, 492, pp.158-164. 10.1016/j.physc.2013.06.009 . hal-00917491

\author{
HAL Id: hal-00917491 \\ https://hal.science/hal-00917491
}

Submitted on 11 Dec 2013

HAL is a multi-disciplinary open access archive for the deposit and dissemination of scientific research documents, whether they are published or not. The documents may come from teaching and research institutions in France or abroad, or from public or private research centers.
L'archive ouverte pluridisciplinaire HAL, est destinée au dépôt et à la diffusion de documents scientifiques de niveau recherche, publiés ou non, émanant des établissements d'enseignement et de recherche français ou étrangers, des laboratoires publics ou privés. 


\title{
Field mapping measurements to determine spatial and field dependence of critical current density in $\mathrm{YBaCuO}$ tapes
}

\author{
J. Leclerc**, K. Berger*, B. Douine*, J. Lévêque* \\ Groupe de Recherche En Electrotechnique de Nancy, University of Lorraine, 54506 \\ Vandoeuvre-les-Nancy, France
}

\begin{abstract}
In this paper a measurement method that allows the determination of the critical current density of superconducting tape from field mapping measurements is presented. This contact-free method allows obtaining characteristics of the superconductor as a function of the position and of the applied flux density. With some modifications, this technique can be used for reel-to-reel measurements. The determination of the critical current density is based on an inverse calculation. This involves calculating the current distribution in the tape from magnetic measurements. An $\mathrm{YBaCuO}$ tape has been characterized at $77 \mathrm{~K}$. A defect in this superconductor has been identified. Various tests were carried out to check the efficiency of the method. The inverse calculation was tested theoretically and experimentally. Comparison with a transport current measurement was also performed.
\end{abstract}

\section{Introduction}

The quality control of superconductors after their fabrication is of high importance for their producers. Particularly, during the fabrication of superconducting tapes of great sizes, it is often the case that a defect is localized on one segment of the tape [1]. The manufacturer has to verify therefore the quality and the capacities of the tapes he produces, before sending them out to the customer. The control method has to be, obviously, a nondestructive one. Characterization methods by field mapping measurements are therefore very suitable. Indeed, these measures are contactless, thus non destructive. In addition, measures acquisition is fast enough for being used in reel-to-reel measurements [2].

\footnotetext{
* Corresponding author

** Principal corresponding author

Email addresses: julien.leclerc@univ-lorraine.fr (J. Leclerc), kevin.berger@univ-lorraine.fr (K. Berger), bruno.douine@univ-lorraine.fr (B.

Douine), jean.leveque@univ-lorraine.fr (J. Lévêque)
} 
Field mapping consists in measuring a map of the flux density produced by a sample. The data obtained by this measurement can be used in different ways. First, simply by viewing the flux density map, inhomogeneities can be identified, signs of defects in the superconductor. Then it is possible to approximate the results by a magnetic field distribution model in order to obtain a value of the critical current density. Finally, by using a technique called "inverse calculation" it is possible to directly visualize the current distribution, and to study how it flows in the superconductor. One advantage of this method is that it allows a spatial characterization of the sample, which is difficult to do with an electrical measurement. In addition, by using a magnet to magnetize the superconductor, it is possible to perform measurements without contact and hence non-destructive. This last feature allows checking the quality of a superconductor before its use.

The field mapping is a measurement technique that has been known for many years. Four main measurement methods have been developed:

- The magneto-optical imaging (MOI) $[3,4,5]$ : it uses a Faraday active film to visualize the value of the magnetic flux density. This film changes the polarization of the light according to the value of the perpendicular component of the imposed flux density. This measurement method is very fast. It provides a good spatial resolution but the magnetic field values obtained are not very accurate (the component of the magnetic field parallel to the film alters the measure [3]).

- The Scanning Hall probe (SHP) [6, 2, 7, 8, 9]: it consists in fixing a Hall effect sensor on a moving table. The sample can be magnetized by an external magnetic field source (permanent magnet or electromagnet). This method provides a good spatial resolution and a high accuracy on the values of the magnetic flux density but is not as fast as the MOI.

- The magnetoscan (MS) $[10,11,12]$ : it is similar to the SHP except that a magnet is fixed directly above the Hall effect sensor. This magnet allows magnetizing the sample. The advantage is that the used magnet is smaller than in the previous method. Nevertheless, it moves with the Hall-effect sensor, which results in a slight variation of the value of the flux density applied to the sample.

- The Hall probe array technique (HPA) $[13,14,15,16,17]$ : An assembly of several Hall effect probes allows to measure the magnetic field simultaneously at several points. This technique is very fast but the spatial resolution is very mediocre because the sensors are separated by about 0.6 $\mathrm{mm}$.

Concerning the magnetization of the superconductor, three methods are possible. The first consists in imposing a transport current to the sample, with or without external applied magnetic flux density $[8,7]$. This method requires 
electrical contacts with the sample. It can therefore damage the superconductor. A second rest on a trapping of magnetic field in the superconductor thanks to a coil or a permanent magnet [9]. A residual imposed flux density can be kept to obtain the field dependence of the characteristics. It is not possible to convert this experiment in a reel-to-reel measurement because the variation of the flux density imposed to the tape is no longer produced by the movement of the tape but by the variation of the field produced by the coil. The tape must stay motionless. A third method consists in cooling the superconducting tape in zero field, and then to apply a flux density. Currents tending to expel the magnetic field then start circulating in the tape. In [14] and [6], the authors use this technique to visualize the distribution of the magnetic field as a function of position across the width of the tape, for different applied flux densities. They approximate the results by a magnetic field distribution model to obtain the value of the critical current density. In [14], Grimaldi et al. achieve to get the value of the critical current density as a function of the position along the length of the tape, but only when the magnetic field is in complete penetration.

We designed an experimental field mapping appartus that is, in our view, the best compromise between speed and accuracy and that allow determining as precisely as possible the critical current density. In addition, this device can be used with some modifications in reel-to-reel measurements. Regarding the measurement method, we chose to use the SHP because it presents a good accuracy in field measuring and spatial positioning while being fast enough to make reel-to-reel measurements [2]. The tape is cooled in zero field and magnetized by permanent magnets. This magnetization process allows applying a constant a constant flux density to the superconducting tape. It has the advantage to not require power supply and can be used in reel-to-reel measurements. Moreover, the value of the applied flux density can be easily changed by varying the distance between the tape and magnets.

We modified the calculation method used in [14] in order to be able to compute the critical current density even when the prenetration of the magnetic field is not complete. This is a key improvement because tapes are often used in these conditions. In addition, the use of SHP rather than HPA, allowed us to obtain a better spatial resolution.

The critical current calculation method is similar to the one used in [18] except that we used a 1D inversion rather the 2D. Indeed, we shown that current densities values obtained by 1D inversion are more accurate (see section 4.2).

In order to check the reliability of the method we inversed the flux density produced by a known current distribution. A comparison of the calculation results with the search current distribution allowed us to evaluate the accuracy of the inverse calculation. Then, a transport current of known value has been imposed to a superconducting tape. After measurement and inversion of the flux density map, we compared the value of the obtained current with the one that was actually circulating in the tape. This step allowed us to verify the effectiveness of the assembly of the measuring apparatus and of the inverse calculation.

The characterization of the tape by our method was then performed. The 


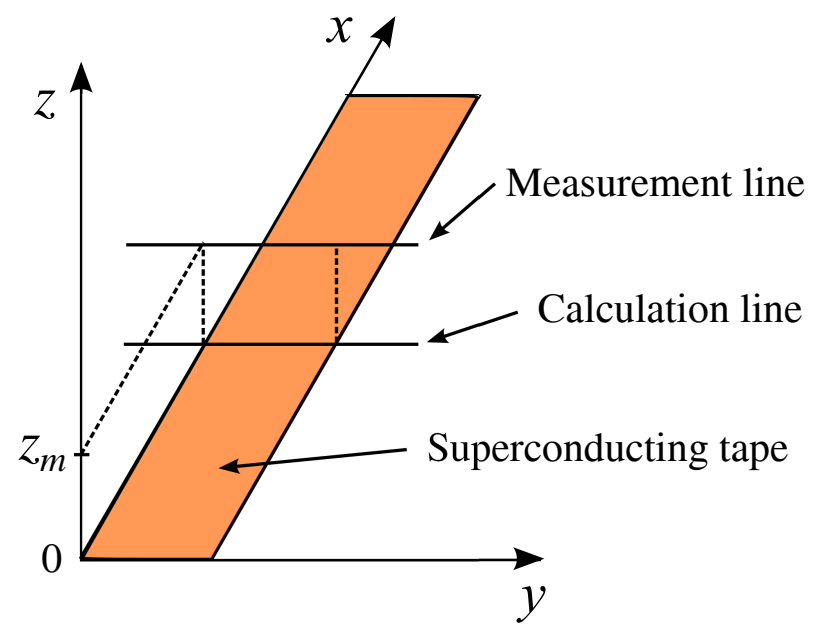

Figure 1: Representation of the measurement layout.

value of the critical current density was thus obtained as a function of the position on the tape and of the flux density. A comparison between these results and those obtained by a 2D inversion has been done in order to validate the assumptions made in the $1 \mathrm{D}$ inversion. Finally, we compared these results with a measurement of the critical current density carried out by the transport current technique in different points of the tape.

\section{Presentation of the method}

\subsection{General description}

The aim of the method is to determine the critical current density of the superconducting tape as a function of the position according to the length of the tape and as a function of the flux density. The superconducting tape is cooled in zero field into liquid nitrogen $(77 \mathrm{~K})$ and then approached to permanent magnets. A map of the flux density of size $m \times n$ is then measured thanks to a SHP device as close as possible above the tape. $m$ and $n$ are respectively the number of points measured in the width and in the length of the tape (Fig. 1). Only the component $B z$ of the flux density perpendicular to the tape is measured. $B z$, represents the sum of the induction $B z_{m}$, produced by the magnets and of the one produced by the tape $B z_{t}$. A $1 \mathrm{D}$ inverse calculation is then performed. For this, the superconducting tape is supposed to be infinitely thin. We therefore consider that it is traversed by a surface current density expressed in $\mathrm{A} / \mathrm{m}$. Each line of measures (Fig. 1) is treated independently. For each line, we dispose of the measurement of $B z$, as a function of $y$. The inverse calculation allows determining, starting from $B z(y)$, the value of $j x$ as a function of $y$, marked as $j x(y)$, with $j x$ being the $x$ component of the current density flowing through the tape. The curve $j x(y)$ is then fitted using a 


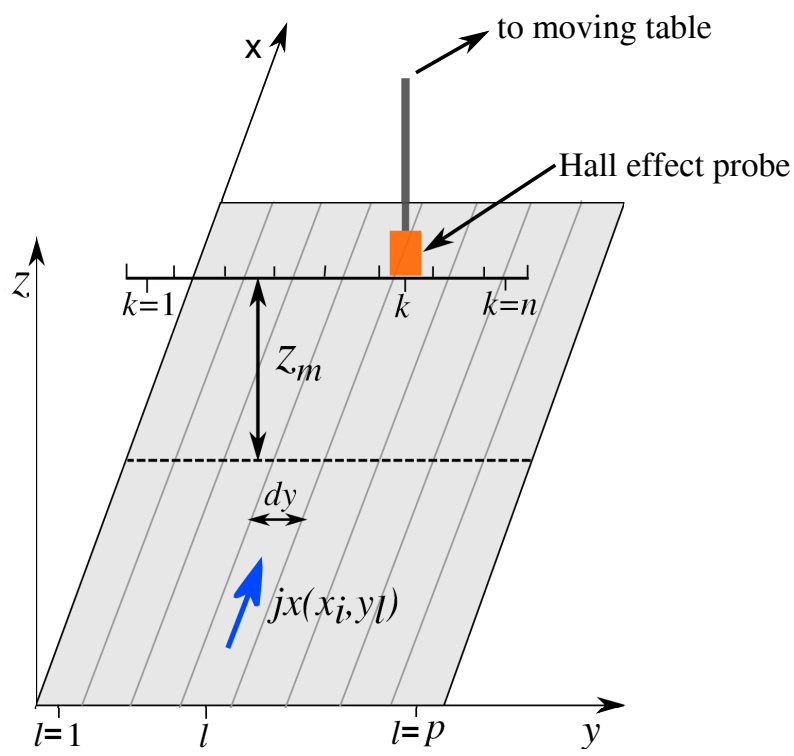

Figure 2: Representation of the grid used for the inverse calculation.

current repartition model (Bean model [19] in the case of full penetration state or Brandt model [20] in the case of incomplete penetration state). $j c$ being a parameter of the model, we obtain a value of $j c$. The operation is repeated for each measurement line. A $j c(x)$ characteristic is thus obtained. By repeating this operation for different applied flux densities (i.e. for different distances between the tape and magnets), the field dependence of the $j c(x)$ characteristic is obtained.

\subsection{The $1 D$ inverse calculation}

The inverse calculation is a very important part of our method. It allows calculating the distribution of currents in the tape, starting from the measurement of the flux density. We used the method described in [21]. The calculation area is divided, according to $y$, in rectangles of width $d y$ and of infinite length, as shown in Fig. 2. We will assume that, in each rectangle, there is a current flowing, which is constant and oriented along $x$. The induction produced by the current circulating in the rectangle located at $y=y_{l}$ and $z=0$ on the point located at $y=y_{k}$ and $z=z_{m}$, can be calculated thanks to the Biot-Savart law:

$$
\begin{gathered}
B z_{t}\left(y_{k}, y_{l}\right)=\frac{j x\left(y_{l}\right)}{10^{7}} \cdot \ln \left(\frac{\left(y_{k}-y_{l}-\frac{d y}{2}\right)^{2}+z_{m}^{2}}{\left(y_{k}-y_{l}+\frac{d y}{2}\right)^{2}+z_{m}^{2}}\right) \\
B z_{t}\left(y_{k}, y_{l}\right)=j x\left(y_{l}\right) \cdot f(k, l)
\end{gathered}
$$

with :

$$
f(k, l)=\frac{1}{10^{7}} \cdot \ln \left(\frac{\left(y_{k}-y_{l}-\frac{d y}{2}\right)^{2}+z_{m}^{2}}{\left(y_{k}-y_{l}+\frac{d y}{2}\right)^{2}+z_{m}^{2}}\right)
$$


The total flux density $B z_{t}$ at the point located at $y=y_{k}$ and $z=z_{m}$ can be calculated thanks to:

$$
B z_{t}\left(y_{k}\right)=\sum_{l=1}^{p} j x\left(y_{l}\right) \cdot f(k, l)
$$

$\mathrm{n}$ and $\mathrm{p}$ are respectively the number of calculation points and the number of measured points. We can write a system on a matrix form:

$$
\begin{gathered}
{\left[\begin{array}{c}
B z_{t}\left(y_{1}\right) \\
B z_{t}\left(y_{2}\right) \\
\ldots \\
B z_{t}\left(y_{n}\right)
\end{array}\right]=\left[\begin{array}{cccc}
f(1,1) & f(1,2) & \ldots & f(1, p) \\
f(2,1) & f(2,2) & \ldots & \ldots \\
\ldots & \ldots & \ldots & \ldots \\
f(n, 1) & \ldots & \ldots & f(n, p)
\end{array}\right]} \\
\mathbf{B} \mathbf{z}_{\mathbf{t}}=\mathbf{F} . \mathbf{j} \mathbf{x}
\end{gathered}
$$

To obtain the repartition of the current in the superconductor, we just have to invert the matrix $\mathbf{F}$ and to multiply it by the vector $\mathbf{B z}_{\mathbf{t}}$, which is built on the basis of the effectuated measurements. $\mathbf{B} \mathbf{z}_{\mathbf{t}}$ is the flux density produced by the tape. It is obtained by subtracting $\mathbf{B} \mathbf{z}_{\mathbf{m}}$ to $\mathbf{B} \mathbf{z}$ where $\mathbf{B} \mathbf{z}_{\mathbf{m}}$ is the flux density produced by the magnets and $\mathbf{B z}$ is the measured flux density. To determine $\mathbf{B} \mathbf{z}_{\mathbf{m}}$ two methods are available to us. The first is to perform a measurement of the flux density without superconducting tape. So the measured field map corresponds to $\mathbf{B} \mathbf{z}_{\mathbf{m}}$. The second solution is to fit $\mathbf{B z}$ by a 5 th order polynomial. Indeed, this polynomial approximates well the edges of the flux density measurements, dominated by the effect of permanent magnets. Despite its relatively high order, it doesn't fit the superconductor effect, at the center of the curve, even when this part is taken into account during the fitting calculations. So, the result obtained by the 5 th order polynomial fit of $\mathbf{B z}$ is very close to $\mathbf{B} \mathbf{z}_{\mathbf{m}}$ (Fig. 3). They are considered equal. We tested both methods and shown that they are equivalent. We chose to use a 5th order approximation. This method is faster because it does not require further measurements.

However, it is not possible to inverse directly $\mathbf{F}$. Indeed, the problem is illposed, and $\mathbf{F}$ is not well conditioned. This is why we have chosen to regularize the system by using the well-known Tikhonov regularization [22,23]. The choice of the regularization parameter was made via the classic method of the plot of the "L curve".

\subsection{Experimental apparatus}

The experimental apparatus (Fig. 4) is composed of three parts: the permanent magnets which impose the magnetic flux density to the superconducting tape, the support for the tape and the flux density measurement system. 5 NdFeB permanent magnets of size of $100 \times 50 \times 10 \mathrm{~mm}^{3}$ each, were placed side by side and installed on an iron plate, to build an assembly with a surface of 500x50 $\mathrm{mm}^{2}$. They allow imposing a flux density varying from 7 to $180 \mathrm{mT}$ to a superconducting tape long of $300 \mathrm{~mm}$, all with an inhomogeneity of less than $3 \%$. The superconducting tape is fixed on a G11 epoxy plate and covered with 


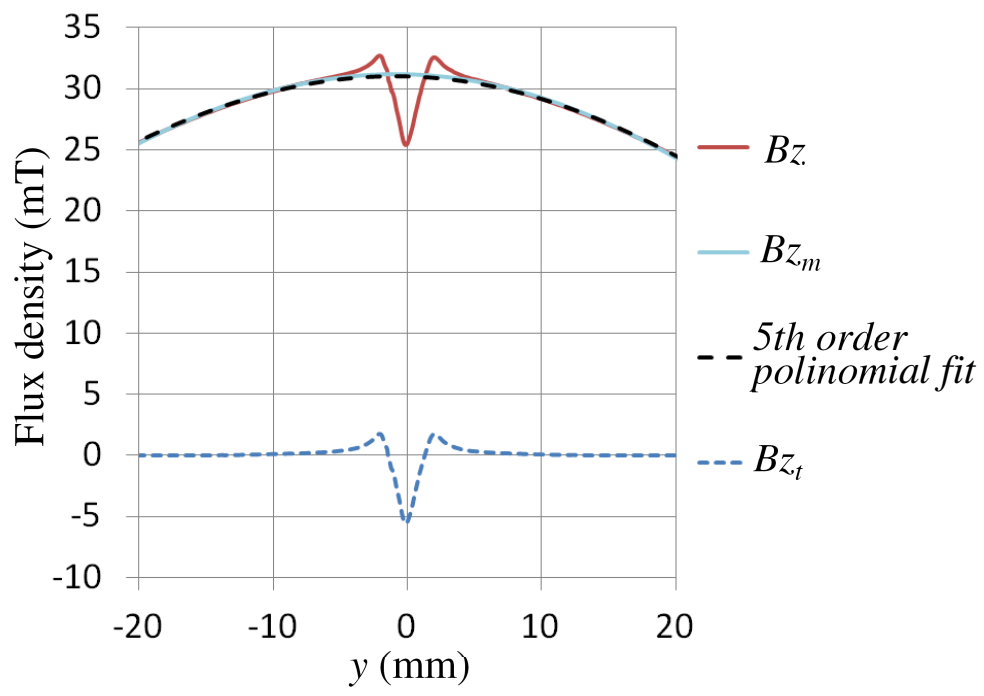

Figure 3: Comparison between the total measured magnetic field $\mathbf{B z}$, the flux density produced by the magnets $\mathbf{B} \mathbf{z}_{\mathbf{m}}$, the fit of $\mathbf{B z}$ by a 5 th order polynomial and the flux density produced by the tape $\mathbf{B} \mathbf{z}_{\mathbf{t}}$. We note that the polynomial and $\mathbf{B} \mathbf{z}_{\mathbf{m}}$ are very close. We consider them equal.

a plastic sheet. Everything can be moved vertically, for varying the distance between the tape and the magnets in order to change the flux density imposed to the tape. The flux density mapping system is, for its part, composed of an Hall effect probe, fixed on a moving table. This system achieves a spatial resolution of $2.5 \mu \mathrm{m}$ and has a flux density precision measurement which is less than $0.1 \mathrm{mT}$. The Hall effect probe is encapsulated in a small Teflon cube. This cube is in contact with the plastic sheet during the measurements. This allows maintaining the $z_{m}$ distance constant.

\section{Validation of the method}

\subsection{Validation of the inverse calculation method}

In order to verify the inverse calculation method, a certain pattern of currents repartition was presupposed in a conductor (Fig. 5). We calculated the flux density which this current should produce, using the Biot-Savart law, at the Hall effect sensor (at an altitude of $z_{m}=420 \mu \mathrm{m}$ ). We added a noise of + or -10 $\mu \mathrm{T}$ to this result. The obtained result, noted as $\mathbf{B} \mathbf{z}_{\mathbf{t h}}$, represents a theoretical measure. The advantage of disposing of a theoretical measure, contrary to the real measure, is that we know precisely the repartition of the current that we aim to determine, by using the inverse calculation method. Hereby, we will be able to check the efficacity of the method. The obtained result by inversing $\mathbf{B} \mathbf{z}_{\mathbf{t h}}$ is presented in Fig. 5. The result is satisfying. As we can notice, the 


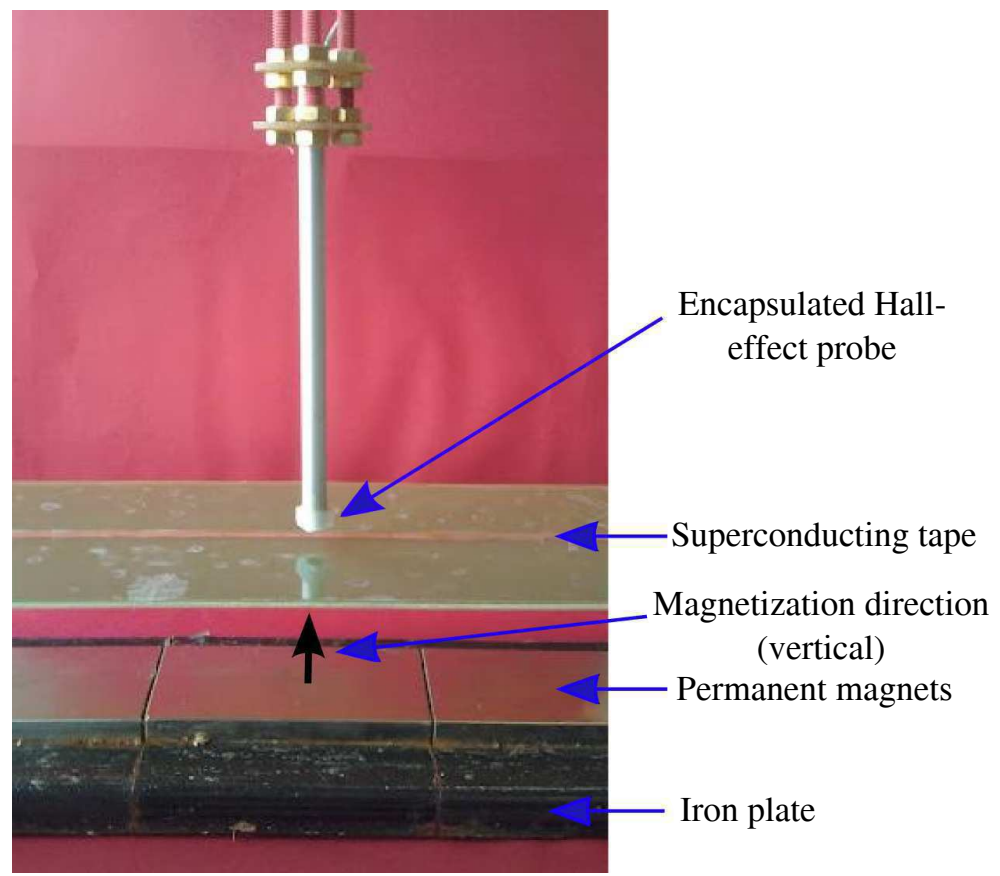

Figure 4: Photograph of the experimental apparatus.

inversed curve corresponds properly to the curve we were searching for. Some undulations appear, due to the regularization.

\subsection{Experimental validation of the method}

The experimental validation of our method was effectuated by imposing a transport current $I_{t r}$ to a superconducting tape, using a power supply. The flux density map was measured with our experimental device and the repartition of the current was calculated with the help of the inverse method. By integrating this result along the width of the tape, we can obtain the value of the current $I_{i n v}$. It is therefore possible to evaluate the good functioning of our method, by comparing $I_{i n v}$ to the value of the current $I_{t r}$, which was effectively flowing through the tape. The experimental tests were made at 20,40,60 and $80 \mathrm{~A}$, at increasing current, and then at 60,40,20 and $0 \mathrm{~A}$, at decreasing current. Results of the field inversion are presented in Fig. 6 .

Firstly, we can note that the repartition of the current is close to the one obtained by the theoretical calculations, in [20]. The shape of the center of the curve is almost identical to the theoretical calculations. However, on the edges, the constants flat parts which should appear are missing. Moreover, the maximal value (or the minimal, in the case of the decreasing current) should remain constant regardless to the current value, which is not our case. In order to verify the values of current obtained with our method, the table 1 allows 


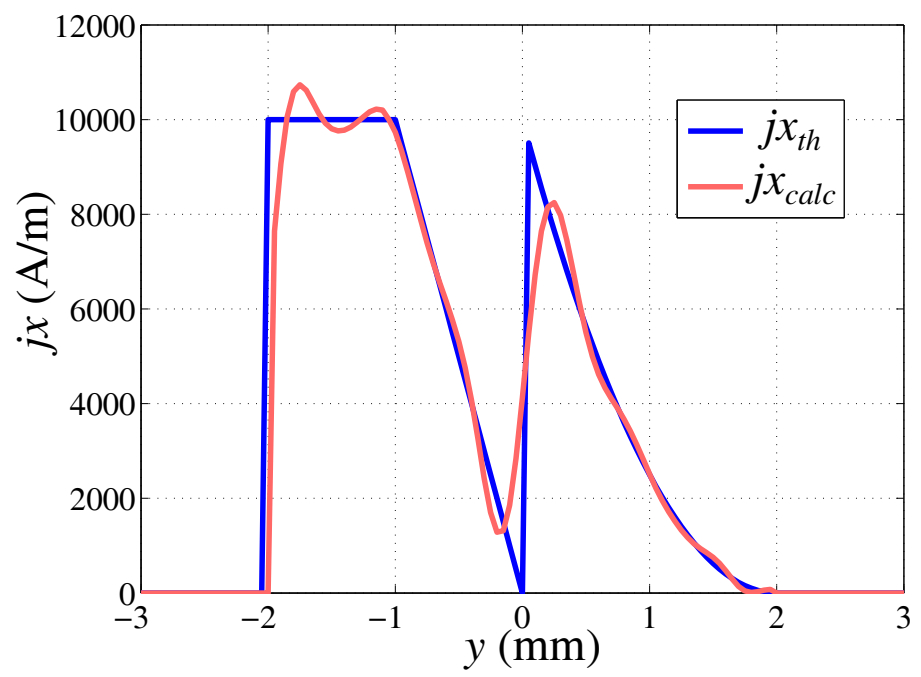

Figure 5: Comparison between the theoretical curve of the current distribution $\left(j_{\text {th }}\right)$ and that obtained by inversion of the flux density map $\left(j_{\text {calc }}\right)$. The $j_{\text {calc }}$ curve corresponds properly to the $j_{t h}$ curve despite some undulations due to the regularization.

Table 1: Comparison between the values of the current imposed experimentaly to the tape and the values values obtained after inversion of the flux density map.

\begin{tabular}{lllll}
\hline & \multicolumn{5}{c}{ Increasing current } \\
\hline Imposed current $I_{t r}(\mathrm{~A})$ & 20.0 & 40.0 & 60.0 & 80.0 \\
Current obtained by inversion $I_{\text {inv }}(\mathrm{A})$ & 17.7 & 35.0 & 53.5 & 73.9 \\
difference (\%) & 11 & 12 & 11 & 7 \\
\hline \multicolumn{5}{c}{ Decreasing current } \\
\hline Imposed current $I_{t r}(\mathrm{~A})$ & 60.0 & 40.0 & 20.0 & 0.00 \\
Current obtained by inversion $I_{i n v}(\mathrm{~A})$ & 56.3 & 38.4 & 19.9 & 1.84 \\
Difference (\%) & 6 & 4 & 0.5 \\
\hline
\end{tabular}

comparing the values of $I_{t r}$ and $I_{i n v}$. We note that the values are close one to another. The biggest difference is of $12 \%$, which is very acceptable. We can therefore conclude that this method allows us to obtain an accurate value of the current flowing through the superconducting tape.

\section{Test of the method}

\subsection{Experimental results}

The method has been tested on an $\mathrm{YBaCuO}$ tape of $30 \mathrm{~cm}$ long, $4 \mathrm{~mm}$ wide and cooled into liquid nitrogen. Measurements were carried out on the $20 \mathrm{~cm}$ located at its center in order to overcome the sides effects (in the inverse calculation, the tape is assumed to be infinite). The resolution in the length direction 


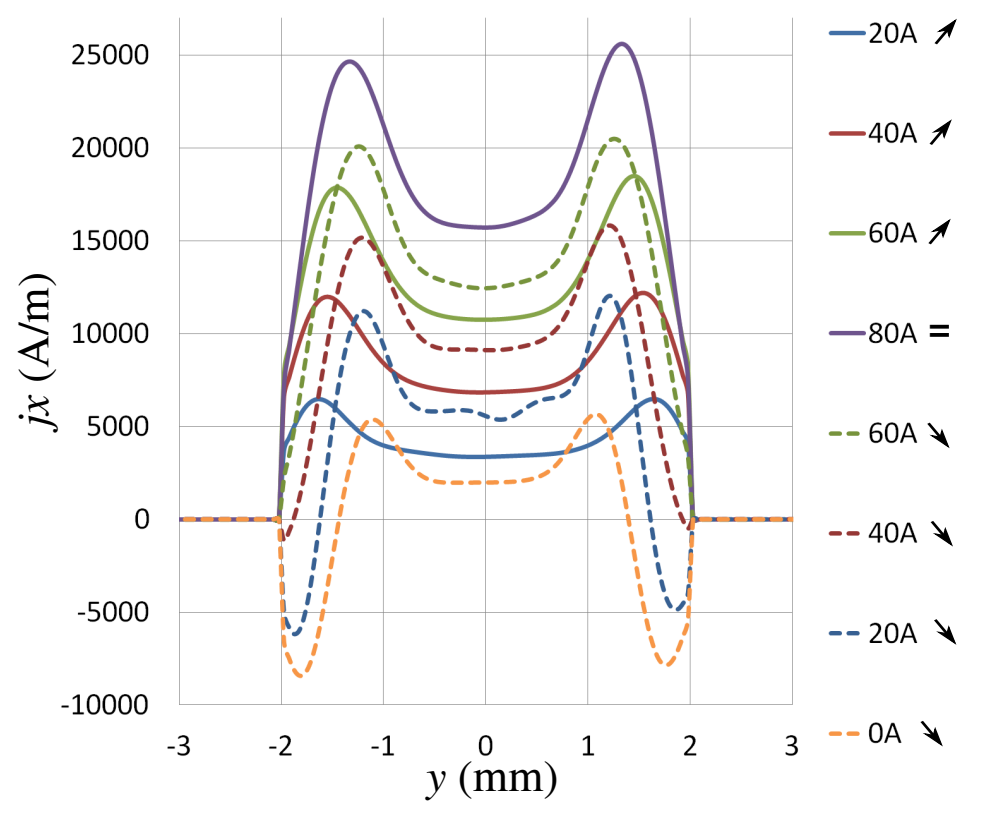

Figure 6: Current distribution $j x$ inside a $\mathrm{YBaCuO}$ tape subject to differents transport currents.

is $1 \mathrm{~cm}$, which corresponds to 21 measurement lines. Each measurement line has a length of $60 \mathrm{~mm}$ with a resolution of $0.1 \mathrm{~mm}$, which corresponds to 601 measurement points per line. Measurements were made for tape-magnets distances equals to $47.5,77.5$ and $107.5 \mathrm{~mm}$, which corresponds to averages imposed flux densities $\left(B_{a p p}\right)$ equals to 38,18 and $10.5 \mathrm{mT}$. This values are the imposed flux densities averaged over the tape width.

The plot of the current distribution for the calculation line located at the middle of the tape is shown in Fig. 7. We can see that for the measurements performed at $B_{a p p}=18$ and $38 \mathrm{mT}$, the current has completely penetrated the superconductor. This is not the case for the measure at $10.5 \mathrm{mT}$ where the current has only partially penetrated.

We also note that the curves obtained have the same shape as those obtained by Brandt, in theoretical calculations [20]. In addition, the current value decreases as the applied magnetic flux density increases. This is due to the decay of the critical current when the flux density increases [24, 25]. Due to the complete penetration of the current, curves at 18 and $38 \mathrm{mT}$ have been fitted by the Bean model. With respect to the curve measured at $10.5 \mathrm{mT}$, it has been approximated by the results of the calculations of Brandt [20], because these ones takes into account the incomplete penetration. Thanks to the good spacial resolution along the tape width, we were able to fit our data with models in a better way than if we had only a few points of measurement (like in HPA). Fits of curves are shown in Fig. 7. This operation was carried out for the 21 


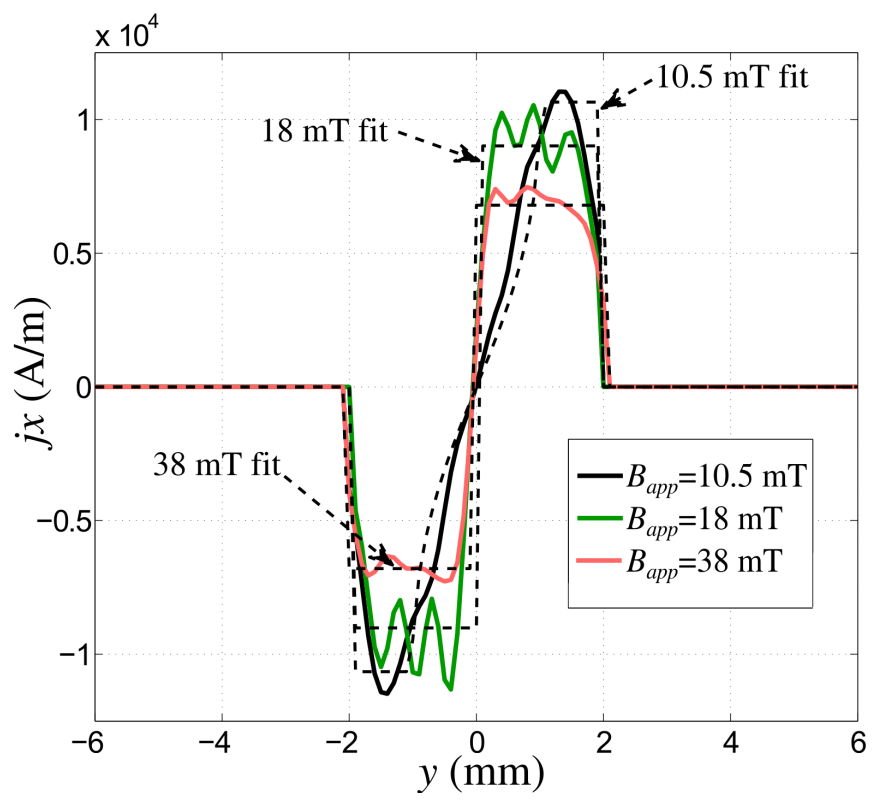

Figure 7: Distribution of the currents induced in the superconducting tape $j x$ by the permanent magnets for different applied flux densities $B_{a p p}$ and their fit using the Brandt model $(10.5 \mathrm{mT})$ and the Bean model (18 and $38 \mathrm{mT})$.

measurement lines. This allowed us to determine $j c(x)$. The results are shown in Fig. 8.

We note that, overall, the critical current density decreases with the increase of the flux density. Some points do not satisfy this assertion, between curves at 10.5 and $18 \mathrm{mT}$. One possible explanation is that the tape does not respond well to the Bean model. Indeed, it is possible that two reactions are opposed. As the flux density is increased, it is plausible that the value of the currents flowing in the tape increases, while penetrating further into the tape. But at the same time, the value of the critical current decreases. So we see a gradual increase of the current at low flux densities, until the decrease of $j c$ becomes predominant and decreases the value of the currents. This explanation is only an assumption and has to be verified.

Moreover, we note that the critical current density strongly decreases at $x=30 \mathrm{~mm}$. This decrease appears very clearly in the three curves. So we are in the presence of a defect in the superconducting tape.

\subsection{Comparison between $1 D$ and $2 D$ inversions}

When the 1D inverse calculation is performed, three assumptions are made: the superconducting tape is infinitely thin, infinitely long and currents circulate only along the $x$ axis. To check the validity of these last two assumptions, we compared the results with those obtained using a 2D inverse calculation. Indeed, 


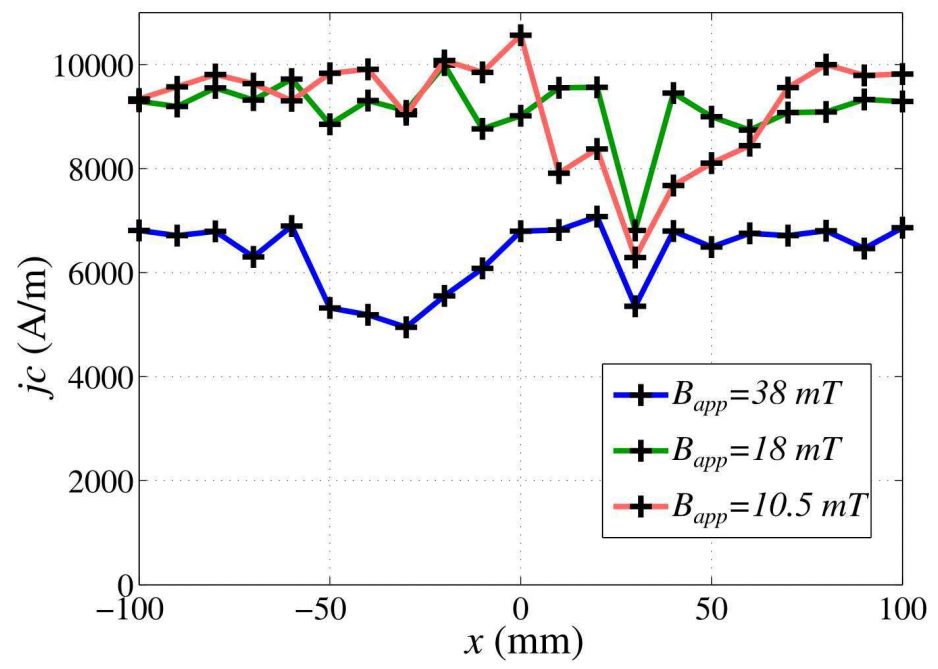

Figure 8: Critical current density $j c$ obtained by field maping, for differents applied flux densities $B_{a p p}$, as a function of the position $x$ on the tape.

for this calculation, the current can flow along the $y$ axis and is of finite length along $x$. The 2D inversion process used is the CG-FFT method described in [26]. For this calculation, it was not necessary to make a regularization. The field mapping measurements of size $21 \times 601$ described in section 3.1 were interpolated to obtain a flux density map of size 300x300. The increase in the number of points along the $x$ axis is required for the $2 \mathrm{D}$ inversion in order to increase the spatial resolution. The decrease of the number of points along the $y$ axis allows to decrease the computation time.

The current density distribution map $j x(x, y)$ obtained by the 2D inversion of the flux density at $10.5 \mathrm{mT}$ is presented Fig. 9. The overall shape of the current distribution is similar to the one that we obtained with the 1D inversion i.e. the current changes direction between each side of the tape. We also note that we can see clearly the defect that we located at $x=30 \mathrm{~mm}$.

The results of this inversion were approximated, as before, to obtain $j c$ as a function of $x$. The $j c(x)$ curves obtained by 2D and 1D inversion are shown in Fig. 10. They have the same shape. The strong decrease of $j c$ at $x=30 \mathrm{~mm}$ is also present in the curve produced by the $2 \mathrm{D}$ inversion. Note however that overall, $j c$ values obtained by this method are lower than with the 1D inversion. With other numerical simulations and experimental tests on known transport currents, we already noticed that the value of currents obtained by 2D inversion were inferior to those we were searching for. This is this problem, which led us to use a $1 \mathrm{D}$ inversion. Indeed, we show that this latter method provides more accurate values. 


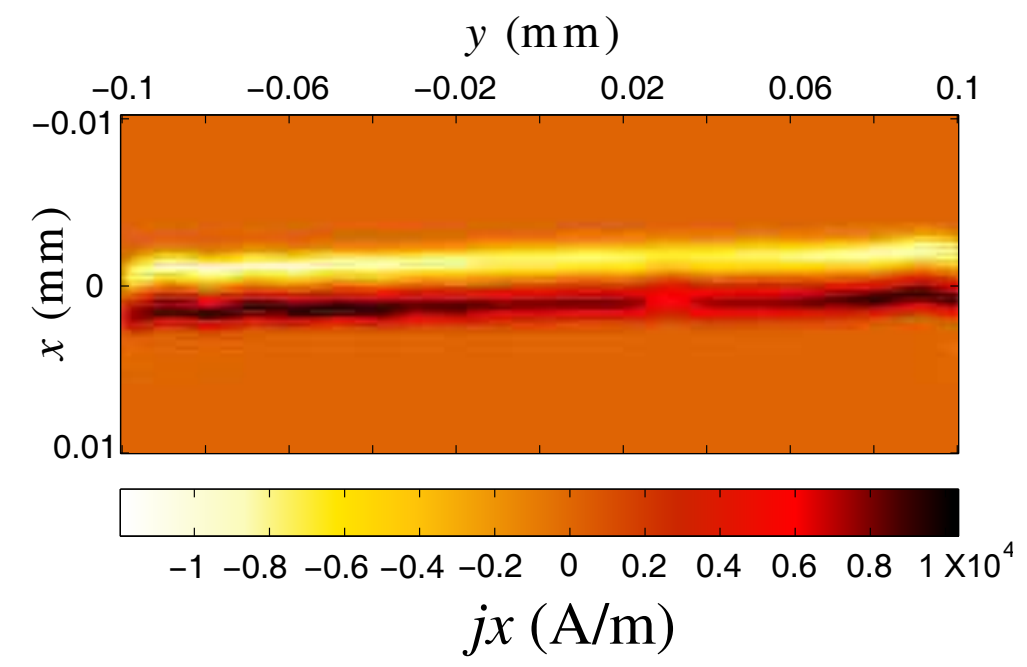

Figure 9: Current distribution map obtained by 2D inversion of the flux density.

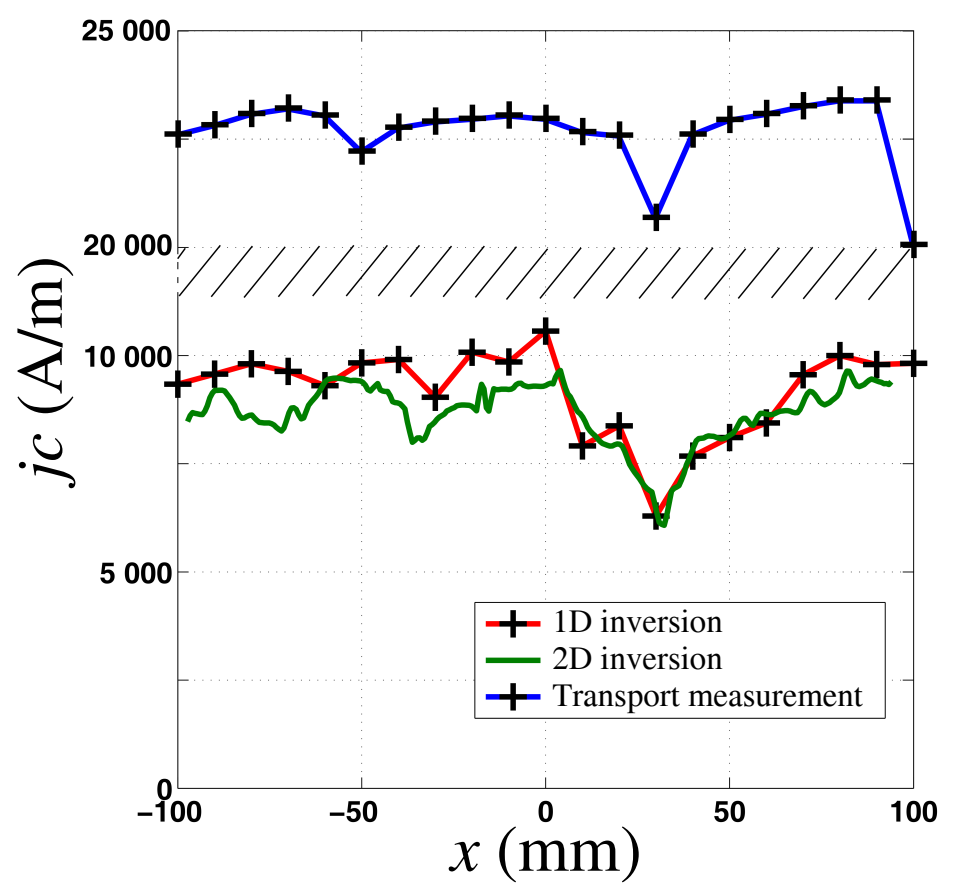

Figure 10: Comparison between critical curent density $j c$ as a function of the position $x$ on the tape obtained by $1 \mathrm{D}$ invesion, 2D inversion and transport measurement.

\subsection{Comparison with the current transport measurement method}

The conventional method for measuring the critical current density of a superconductor is the transport current method. We performed measurements 


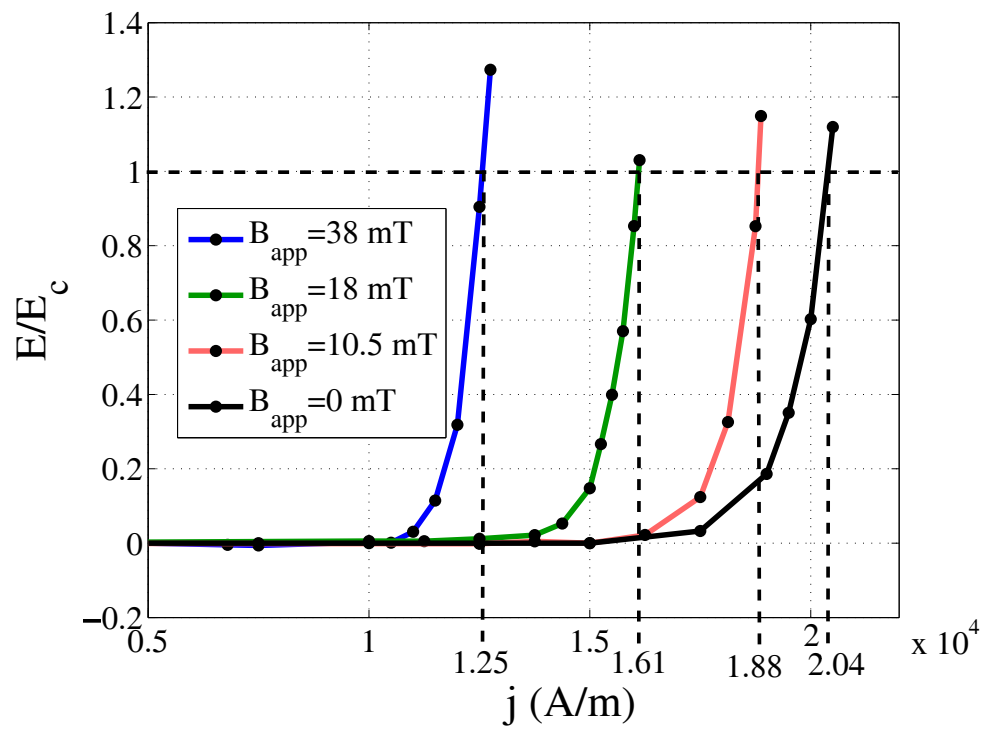

Figure 11: Normalized electric field $E / E c$ as a fuction of the current density $j x$ for differents applied flux densities $B_{a p p}$. These curves were measured by the current transport method.

by this method to verify the results obtained by field mapping. The critical current density has been determined using the $1 \mu \mathrm{V} / \mathrm{cm}$ standard criterion . The results obtained by measuring the $20 \mathrm{~cm}$ of tape previously tested are presented Fig. 11. We note that the critical current density obtained is greatly superior to that we obtained by field mapping.

We also carried transport measurements of $j c$ as a function of the position on the tape by moving 2 voltage leads spaced from $1 \mathrm{~cm}$ along the superconductor. The electrical contact is ensured here by pressure of the voltage leads. These last measurements were performed in self field. The $j c(x)$ curve (Fig. 10) shows once again that the values are much higher than those obtained by field mapping. We also note that the previously identified defect appears and is located in the same position. This proves that our field mapping apparatus effectively detect defects. We also note that at the end of the tape (at $x=100 \mathrm{~mm}$ ) the critical current density decreases. This is due to the welding of a voltage lead that we have made to perform the measurements of Fig. 11. The heat generated by the welding process has degraded the properties of the tape.

The superiors $j c$ values obtained by transport measurements can be explained by a difference of criterion between the two methods. Indeed, we assumed that the behavior of the superconductor corresponds to the Bean model (Brandt calculations are based on the Bean model), but this is not exactly the case. Superconductors obey to a power law (7) and high temperature superconductors have a relatively low $\mathrm{n}$ exponent. However the Bean model corresponds to an infinite $\mathrm{n}$ value. The critical current density is usually defined as the 
current density required to obtain an electric field of $1 \mu \mathrm{V} / \mathrm{cm}$. This value is an arbitrary criterion. Nevertheless, in our new method, we are never considering this criterion. The definition of the critical electric field of our method is rather "the maximum electric field that allows the current flowing through the tape for a few hours (time of measurement) without observable attenuation". This difference of critical electric field gives, according to the power law, a difference in critical current values.

$$
\frac{E}{E c}=\left(\frac{j}{j c}\right)^{n}
$$

\section{Conclusion}

A field mapping apparatus for measuring the critical current of superconducting tapes has been developed and implemented. This method, in addition to being contactless, allows measuring the critical current density as a function of the position on the tape for different applied magnetic fields. It is based on the measurement of a map of the flux density and on an inverse calculation. Permanent magnets were used in order to induce currents in the superconductor.

We made several tests to check the efficiency of the method. First we inversed the flux density produced by a known current. A comparison of the calculation results with the search current distribution allowed us to evaluate the accuracy of the inverse calculation. Then we imposed a transport current of known value to a superconducting tape. After measurement and inversion of the flux density map, we compared the value of the obtained current with the one that was actually circulating in the tape. In addition, a comparison between 1D and 2D inversions was made.

A superconducting $\mathrm{YBaCuO}$ tape was characterized at $77 \mathrm{~K}$ by this method. The value of the critical current density was obtained as a function of the position on the tape for three values of external applied flux densities. A defective area having a lower critical current density has been detected.

A comparison with the conventional transport current method was performed. The default localized by the field mapping method also appears in these measures. However, the value of the critical current density obtained is very different. This is due to a different critical electric field between the two methods.

\section{References}

[1] G. Kastner, D. Hesse, R. Scholz, H. Koch, F. Ludwig, M. Lorenz, and H. Kittel. Microstructure defects in $Y B C O$ thin films: A TEM study to discuss their influence on device properties. Physica C: Superconductivity, 243(3-4):281 - 293, 1995. 
[2] Kohei Higashikawa, Kei Shiohara, Yoshihiro Komaki, Keitaro Okumura, Kazutaka Imamura, Masayoshi Inoue, Takanobu Kiss, Yasuhiro Iijima, Takashi Saitoh, Takato Machi, Masateru Yoshizumi, Teruo Izumi, and Hiroshi Okamoto. High-speed scanning hall-probe microscopy for twodimensional characterization of local critical current density in long-length coated conductor. Physics Procedia, 27(0):228 - 231, 2012. Proceedings of the 24th International Symposium on Superconductivity (ISS 2011), October 24-26, 2011, Tokyo, Japan.

[3] T. Johansen, M. Baziljevich, H. Bratsberg, Y. Galperin, P. Lindelof, Y. Shen, and P. Vase. Direct observation of the current distribution in thin superconducting strips using magneto-optic imaging. Physical Review B, 54(22):16264-16269, December 1996.

[4] Rinke Wijngaarden, H. Spoelder, R. Surdeanu, and R. Griessen. Determination of two-dimensional current patterns in flat superconductors from magneto-optical measurements: An efficient inversion scheme. Physical Review B, 54(9):6742-6749, September 1996.

[5] Ch Jooss, J Albrecht, H Kuhn, S Leonhardt, and H Kronmuller. Magnetooptical studies of current distributions in high- Tc superconductors. Reports on Progress in Physics, 65(5):651-788, May 2002.

[6] Jaeun Yoo, Yonghwan Jung, Jaeyoung Lee, Sunme Lim, SangMoo Lee, YeHyun Jung, Dojun Youm, Hosup Kim, HongSoo Ha, and Sangsoo Oh. Scanning hall probe measurements of field distributions of a coated conductor under applied fields. Superconductor Science and Technology, 19(12):1291-1296, December 2006.

[7] M. Carrera, J. Amoros, X. Granados, R. Maynou, T. Puig, and $\mathrm{X}$. Obradors. Computation of current distribution in ybco tapes with defects obtained from hall magnetic mapping by inverse problem solution. Applied Superconductivity, IEEE Transactions on, 21(3):3408 -3412, june 2011.

[8] M. Polak, P.N. Barnes, P. Mozola, and G.A. Levin. Critical current in ybco coated conductors in the presence of a macroscopic defect. Applied Superconductivity, IEEE Transactions on, 19(3):2921 -2924, june 2009.

[9] K. Higashikawa, M. Inoue, T. Kawaguchi, K. Shiohara, K. Imamura, T. Kiss, Y. Iijima, K. Kakimoto, T. Saitoh, and T. Izumi. Scanning hallprobe microscopy system for two-dimensional imaging of critical current density in re-123 coated conductors. Physica C: Superconductivity, 471(2122):1036 - 1040, 2011. The 23rd International Symposium on Superconductivity.

[10] F Hengstberger, M Eisterer, M Zehetmayer, and H W Weber. Assessing the spatial and field dependence of the critical current density in ybco 
bulk superconductors by scanning hall probes. Superconductor Science and Technology, 22(2):025011, 2009.

[11] M. Zehetmayer, R. Fuger, M. Eisterer, F. Hengstberger, and H. W. Weber. Assessment of the local supercurrent densities in long superconducting coated conductors. APPLIED PHYSICS LETTERS, 90(3), JAN 152007.

[12] F. Hengstberger, R. Fuger, M. Eisterer, H.W. Weber, and D. Isfort. Homogeneity of supercurrent flow in coated conductors. Physica C: Superconductivity, 460 - 462, Part 2(0):1397 - 1398, 2007.

[13] Ho-Sup Kim, Sang-Soo Oh, Nam-Jin Lee, Seung-Kyu Baik, Dong-Woo Ha, Rock-Kil Ko, Hong-Soo Ha, Tae-Hyung Kim, Do-Jun Youm, Seung hyun Moon, and Chan Park. Nondestructive measurement of critical current distribution of smbco coated conductor using hall probe. Applied Superconductivity, IEEE Transactions on, 20(3):1537 -1540, june 2010.

[14] G Grimaldi, M Bauer, and H Kinder. Continuous reel-to-reel measurement of critical currents of coated conductors. APPLIED PHYSICS LETTERS, 79(26):4390-4392, DEC 242001.

[15] S Furtner, R Nemetschek, R Semerad, G Sigl, and W Prusseit. Reel-to-reel critical current measurement of coated conductors. Superconductor Science and Technology, 17(5):S281, 2004.

[16] G Grimaldi, M Bauer, H Kinder, W Prusseit, U Gambardella, and S Pace. Magnetic imaging of $Y B C O$ coated conductors by hall probes. Physica $C$ : Superconductivity, 372 - 376, Part 2(0):1009 - 1011, 2002.

[17] Theva | Tapestar. http://www.theva.com/prod/tapestar.

[18] J Amoros, M Carrera, and X Granados. An effective model for fast computation of current distribution in operating $H T S$ tapes from magnetic field measurements in non-destructive testing. Superconductor Science and Technology, 25(10):104005, 2012.

[19] C. P. Bean. Magnetization of hard superconductors. Phys. Rev. Lett., 8:250-253, Mar 1962.

[20] Ernst Helmut Brandt and Mikhail Indenbom. Type-2-superconductor strip with current in a perpendicular magnetic field. Phys. Rev. B, 48:1289312906, Nov 1993.

[21] T. H. Johansen, M. Baziljevich, H. Bratsberg, Y. Galperin, P. E. Lindelof, Y. Shen, and P. Vase. Direct observation of the current distribution in thin superconducting strips using magneto-optic imaging. Phys. Rev. B, 54:16264-16269, Dec 1996.

[22] G. Golub, P. Hansen, and D. O'Leary. Tikhonov regularization and total least squares. SIAM Journal on Matrix Analysis and Applications, 21(1):185-194, 1999. 
[23] Rainer Kress, Lars Kuhn, and Roland Potthast. Reconstruction of a current distribution from its magnetic field. Inverse Problems, 18(4):1127, 2002.

[24] Y. B. Kim, C. F. Hempstead, and A. R. Strnad. Critical persistent currents in hard superconductors. Phys. Rev. Lett., 9:306-309, Oct 1962.

[25] J. Leclerc, K. Berger, B. Douine, and J. Leveque. Enhancement of the $E(J, B)$ power law characterization for superconducting wires from electrical measurements on a coil. Applied Superconductivity, IEEE Transactions on, 22(3):6400504-6400504, 2012.

[26] Rinke J. Wijngaarden, K. Heeck, H.J.W. Spoelder, R. Surdeanu, and R. Griessen. Fast determination of $2 D$ current patterns in flat conductors from measurement of their magnetic field. Physica C: Superconductivity, 295(3-4):177 - 185, 1998. 